Tohoku J. Exp. Med., 2008, 216, 133-137

\title{
Nonrecurrent Inferior Laryngeal Nerve Without Vascular Anomaly as a Genuine Entity
}

\author{
Masaru Tateda, ${ }^{1}$ Jun Hasegawa, ${ }^{2}$ Shun Sagai, ${ }^{2}$ Ayako Nakanome, ${ }^{2}$ \\ Katsunori Katagiri, ${ }^{1}$ Eitchi Ishida, ${ }^{3}$ Ryuzo Kanno, ${ }^{4}$ Takeo Hasegawa ${ }^{4}$ \\ and TOSHIMITSU KOBAYASHI ${ }^{2}$ \\ ${ }^{1}$ Department of Otolaryngology, Iwate Medical University School of Medicine, Morioka, Japan \\ ${ }^{2}$ Department of Otolaryngology-Head \& Neck Surgery, Tohoku University Hospital, Sendai, Japan \\ ${ }^{3}$ Department of Otolaryngology, Iwaki Kyoritsu General Hospital, Iwaki, Japan \\ ${ }^{4}$ Department of Thoracic Surgery, Iwaki Kyoritsu General Hospital, Iwaki, Japan
}

Inferior laryngeal nerve (ILN), which usually arises from vagus nerve, runs recurrent course and is called recurrent ILN (RILN). Intimate knowledge of normal and anatomic variants of the ILN reduces the risk of nerve injury and vocal cord paralysis in thyroid and parathyroid surgery. The nonrecurrent ILN (NRILN) is a rare nerve anomaly that is associated with a right aberrant subclavian artery. We encountered 2 patients with NRILN during thyroid surgery. Patient 1, a 57-year-old woman, had mediastinal thyroid tumor without vascular anomaly. Patient 2, a 47-year-old woman with a history of esophageal foreign body (fish bone), was suspected to have NRILN with vascular anomaly before surgery, as judged by preoperative enhanced CT. In the patient 1 , we verified laryngeal mobility function of the NRILN by nerve stimulation, and confirmed the absence of RILN in usual recurrent course. The findings in the patient 1 indicate that NRILN without subclavian artery anomaly is a genuine entity. In both patients we performed thyroid surgery safely and fast by expectation and identification of NRILN by use of nerve stimulator. In conclusion, we confirm the existence of NRILN without vascular anomaly and show effectiveness of preoperative enhanced CT and valuable use of nerve stimulator for nerve preservation in the patients with NRILN. —— Nonrecurrent inferior laryngeal nerve; aberrant subclavian artery; esophageal foreign body; nerve stimulator; CT.

Tohoku J. Exp. Med., 2008, 216 (2), 133-137.

(C) 2008 Tohoku University Medical Press

Inferior laryngeal nerve (ILN) palsy is a major problem in thyroid and parathyroid surgery. Intimate knowledge of normal and anatomic variants of the ILN reduces the risk of nerve injury and vocal cord paralysis. It is generally accepted that the nonrecurrent inferior laryngeal nerve (NRILN) is a well-known but relatively rare anatomic variant. The prevalence of NRILN is less than $1 \%$ based on surgical reports (Henry et al. 1988; Proye et al. 1991; Avisse et al. 1998; Devèze et al. 2003; Toniate et al. 2004) and 1-2\% based on anatomical reports (Avisse et al. 1998). The NRILN usually arises on the right side of the neck and is associated with malformation of the aortic arch and aberrant subclavian artery. In NRILN, there are several unsolved problems,

Received April 15, 2008; revision accepted for publication August 27, 2008.

Correspondence: Masaru Tateda M.D., Department of Otolaryngology, Iwate Medical University School of Medicine, 19-1 Uchimaru, Morioka, Iwate 020-8505, Japan.

e-mail: tatedam@iwate-med.ac.jp 
such as the patients without vascular anomaly, necessity of preoperative diagnosis of vascular anomaly by imaging and use of nerve stimulator for identify of ILN. We encountered 2 NRILNs during thyroid surgery at Iwaki Kyoritsu General Hospital. One had no vascular anomaly, while the other had a vascular anomaly. We describe these patients and review the related literature. This study was approved by the ethics committee of Iwaki Kyoritsu General Hospital.

\section{Clinical Findings}

\section{Patient 1}

A 57-year-old woman underwent chest X-ray as part of a periodic medical examination, revealing an abnormal opacity in the right superior mediastinum. Enlargement of a thyroid tumor into the superior mediastinum was detected on CT (Fig. 1). The patient had undergone surgery on the right side of her thyroid 30 years previously at

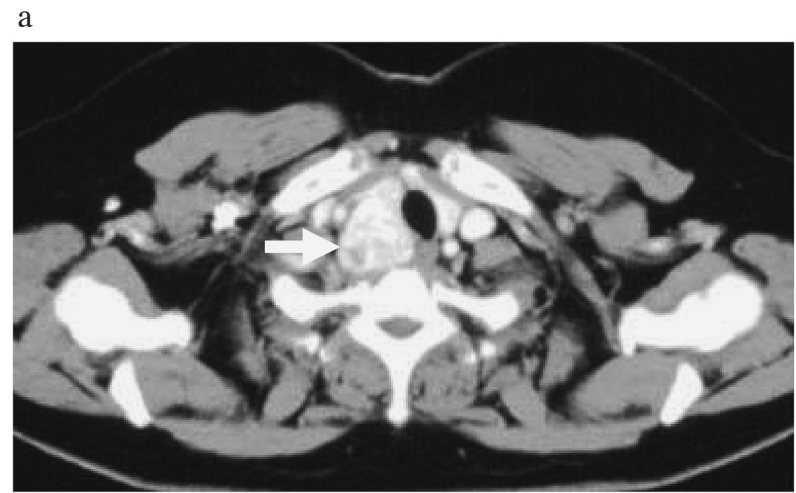

b

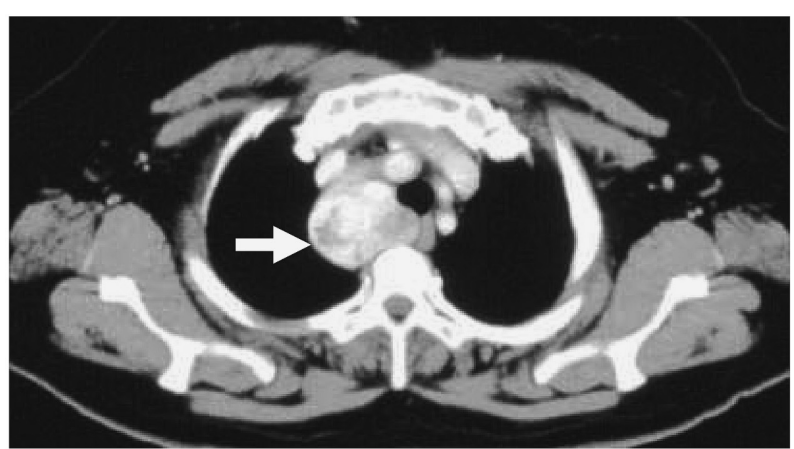

Fig. 1. Enhanced CT image of patient 1. a; A tumor was apparent in the right thyroid gland (arrow). $\mathrm{b}$; The tumor had enlarged into the superior mediastinum (arrow).

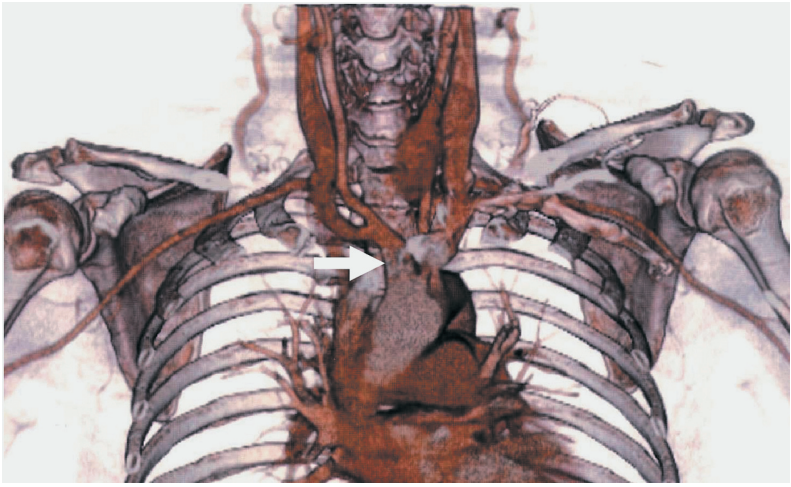

Fig. 2. Enhanced CT image with 3D reconstruction of patient 1 . The right subclavian artery arises from the brachiocephalic artery (arrow).

another institution. She had experienced no symptoms such as cervical swelling, dysphasia, or hoarseness, and ILN palsy was not evident from endoscopic examination. During surgery, the recurrent ILN (RILN) could not be identified due to the massive tumor. Therefore, we could not approach its usual location near the inferior thyroid artery. We dissected carefully around where the ILN enters the larynx and identified the ILN superiorly by nerve stimulator monitoring. The tumor was removed via the cervical approach alone and was found to be impinging on the ILN inferiorly. When the tumor was finally removed superiorly, we found that the ILN was nonrecurrent and arose directly from the vagus nerve. The NRILN was preserved and its function was confirmed by nerve stimulation during surgery. Postoperative fiberscopic examination of the vocal cords showed normal function. No aberrant subclavian artery was detected on postoperative enhanced CT (Fig. 2).

\section{Patient 2}

A 47-year-old woman had a history of esophageal foreign body (fish bone), with tracheostomy having been performed 2 years previously. The tracheostoma was maintained at followup visits. Right thyroid tumor was detected on follow-up CT performed for the evaluation of tracheal stenosis. Fine needle aspiration biopsy under ultrasonography suggested papillary carcinoma. Preoperative enhanced CT showed an 
a

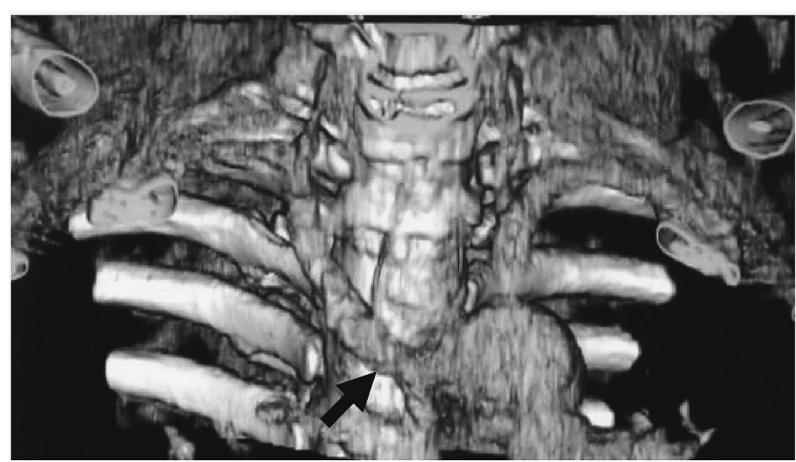

b

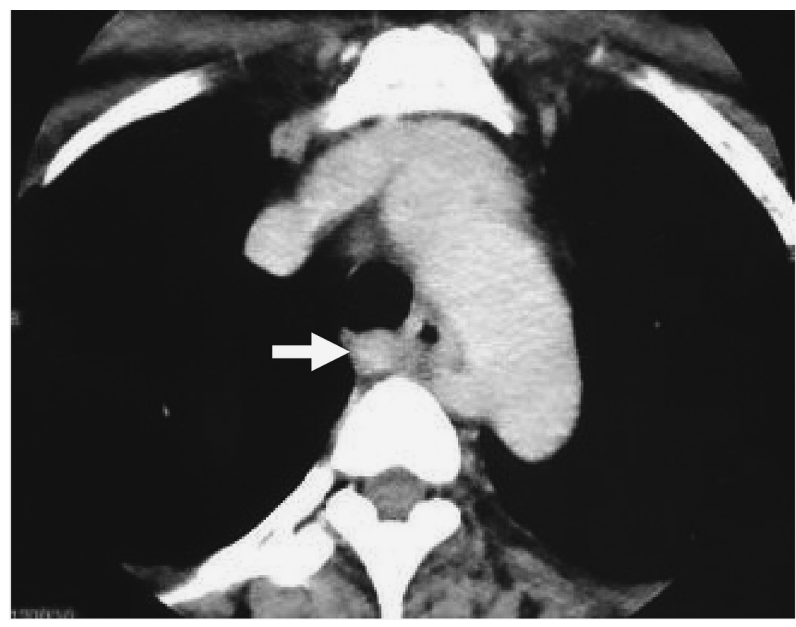

Fig. 3. Enhanced CT image of patient 2. a; CT image with reconstruction. The right subclavian artery arises from the aortic arch (arrow). b; It passes laterally behind the esophagus and trachea (arrow).

aberrant subclavian artery (Fig. 3). We operated carefully using a nerve stimulator and, as expected, found an NRILN that crossed directly from the vagus nerve and passed transversely across the neck. Vocal cord function after surgery remained normal. Postoperative barium swallow test revealed a posterior notch indicating an aberrant subclavian artery, the so-called bayonet sign (Fig. 4).

\section{Discussion}

The first anatomical case with right aberrant subclavian artery was described by Hunauld in 1785, but the first clinical case of retroesophageal right subclavian artery was described in 1794 by

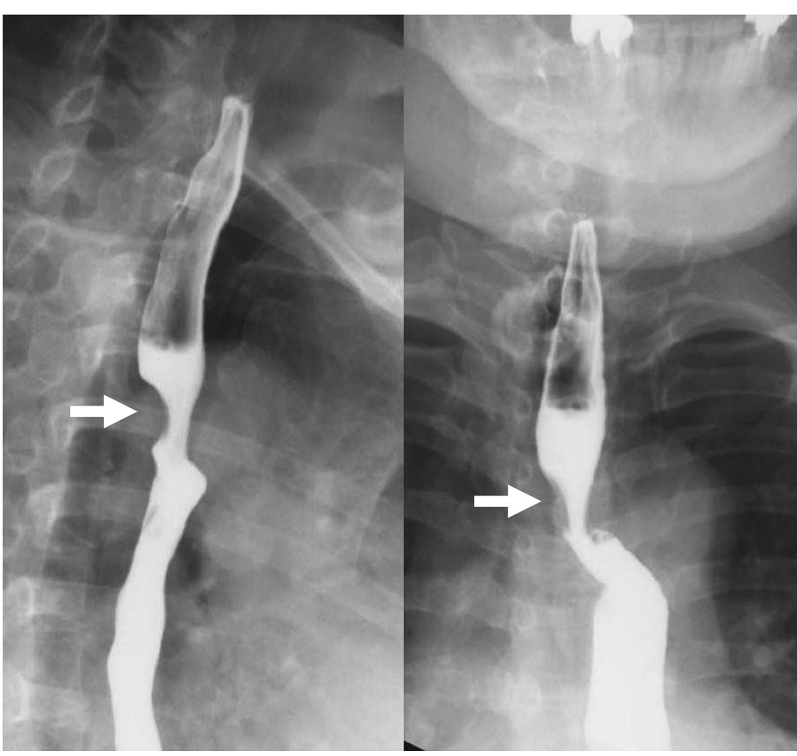

Fig. 4. Barium swallow image of patient 2. Notch mark indicates an aberrant retropharyngeal right subclavian artery (arrow).

Bayford in a patient who suffered from dysphagia: the term "dysphagia lusoria" has been used to describe this symptomatic consequence of the vascular anomaly (Henry et al. 1988; Avisse et al. 1998). Arkin naturally named the abnormal artery arteria lusoria (Henry et al. 1988). The neural anomaly was identified in 1823 (Stedman 1823), but its surgical interest was pointed out only in 1932 (Pemberton and Beaver 1932). The formation of a NRILN is attributed to abnormal embryological development of the subclavian artery. In the embryo, the right and left ILNs supply their respective sixth branchial arches. As the embryo elongates and the heart descends, the nerves assume their recurrent course, wrapping around the sixth arches bilaterally. On the right, the distal sixth arch disappears and the right ILN is free to ascend as high as the subclavian artery (the fourth aortic arch). On the left, the sixth aortic arch remains until birth as the ductus arteriosus, and later as the ligamentum arteriosum. If the fourth aortic arch disappears, the ILN reaches the larynx directly, resulting in a NRILN. In most instances, the right subclavian artery arises from the distal portion of the left subclavian artery and passes to the right behind the esophagus, becoming an aberrant subclavian artery (Gray et al. 1976; 
Nagayama et al. 1994). The presence of left NRILN is extremely rare and is always associated with situs inversus viscerum. Only four patients with left NRILN have been reported (Henry et al. 1988; Coady et al. 2000; Fellmer at al. 2008). Kobayashi reported that patients with aberrant subclavian artery tend to have multiple vascular anomalies and ischemic vascular disease. Therefore if a nerve variant such as NRILN is found during surgery, subsequent investigation of vascular abnormalities may help to prevent unexpected life-threatening events (Kobayashi et al. 2007).

In patient 1 , we unexpectedly found a NRILN intraoperatively. No aberrant subclavian artery was found on enhanced 3D CT. Although the patient had undergone a thyroid operation 30 years earlier, she had not previously had vascular surgery or laryngeal nerve surgery. She experienced no symptoms related to the previous thyroid operation such as hoarseness, and no symptoms related to an aberrant subclavian artery such as dysphagia, cough, stridor, or thoracic pain before the first thyroid operation. Five cases of NRILN without vascular anomaly have been reported (Henry et al. 1988; Kato et al. 1996; Coady et al. 2000). In 4 of these patients, the NRILN was accompanied by an ipsilateral recurrent ILN (RILN). The authors of the remaining report did not describe a coexisting RILN, and they reported the absence of situs inversus and right aortic arch (Coady et al. 2000). There is no appropriate embryological and anatomical reason to explain existence of NRILN without vascular anomaly. Therefore, NRILN in the patients without vascular anomaly has been thought to be a different nerve, which was mistaken for NRILN by its running course (Raffaelli et al. 2000; Maranillo et al. 2008). Raffaelli described the false NRILN, which is an anastomotic nerve (AN) between the sympathetic nerve (SN) and RILN. It is usually thin, sometimes having the same diameter as the RILN (Raffaelli et al. 2000). Raffaelli mentioned that, if a large AN is discovered, it may be mistaken for a NRILN. Incidence of large AN confined to the right side is $1.5 \%$ based on surgical reports. However, based on anatomical reports, Maranillo found that incidence of large AN was $17.5 \%$ on the right side and $17 \%$ on the left side (Maranillo et al. 2008). In the present patients we could not find coexisting RILN. In patient 1 , if the laryngeal nerve was a branch of the vagus nerve it would have only the role of mobilizing the vocal cords. We tested mobility function of the NRILN by a nerve stimulator, and the results suggested that this was a genuine NRILN. But if this nerve was large AN i.e. "false NRILN", the fact that it was derived from the $\mathrm{SN}$ means that the $\mathrm{SN}$ was involved in vocal cord mobility. The issue of whether the SN is involved in vocal cord mobility is very important for surgeons during head and neck surgery. In experimental studies it has been suggested that possible roles of the $\mathrm{SN}$ in vocal cord function are the regulation of glandular secretion and vasculization (Basterra et al. 1989), but the SN has not yet been found to control movement of the vocal cords. The accurate role of sympathetic fibers of the AN has not yet been clarified. Therefore, at present we must preserve not only the RILN and NRILN, but also any branch of the SN to avoid vocal cord paralysis.

Patient 2 had a history of esophageal foreign body (tuna fish bone). Tracheostomy had been performed due to pharyngoesophageal injury by the bone. After decannulation of the tracheostomy, we attempted to close the tracheostoma twice; however, dyspnea due to tracheal stenosis appeared a few weeks later, and re-tracheostomy was required each time. Reconstructive surgery was not possible due to mental illness. We therefore left the tracheostoma patent. The patient had an aberrant subclavian artery, a so-called arteria lusoria, which was confirmed on preoperative enhanced CT and barium swallow. Dysphagia is the main symptom of arteria lusoria. Several preoperative diagnostic investigations for vascular anomaly have been described, including CT, barium swallow, and ultrasonography (Nagayama et al. 1994; Watanabe et al. 2001; Hermans et al. 2003, Devèze et al. 2003). In this patient, evaluation of preoperative CT including the neck to mediastinum was very useful and enabled the operation to be performed safely and fast. 
Although necessity of preoperative CT for all patients with thyroid or parathyroid tumor is controversial, we thought that information of vascular anomaly by CT or ultrasonography is necessary for safely and fast operation to avoid nerve injury.

A search of the literature revealed no patients of vascular anomaly in a patient with history of esophageal foreign body, and no reports of NRILN in these circumstances. The relationship between vascular anomalies and foreign body remains unclear. Because the tracheostoma was at a different level to the vascular anomaly, we consider that difficulty with decannulation was unrelated to the vascular anomaly.

Although one study found that routine application of nerve monitoring techniques is not recommended (Chan and Lo 2006), these techniques are very useful in patients such as those presented here, and they help to avoid nerve injury. We recommend using a nerve stimulator to avoid nerve injury when a NRILN is predicted preoperatively, when it is anticipated that the RILN will be hard to identify (for example, in mediastinal thyroid tumor), and when the RILN is unexpectedly difficult to find.

We encountered 2 NRILNs, one with and one without vascular anomaly. The existence of a NRILN without vascular anomaly is very rare and remains controversial. We show that NRILN without aberrant subclavian artery is a genuine entity. We confirmed effectiveness of preoperative enhanced CT and valuable use of nerve stimulator in the patients with NRILN. To avoid nerve injury, thyroid surgeons must always be prepared to encounter the NRILN with various other anomalies, with other vascular disease, and with unknown anomalies.

\section{References}

Avisse, C., Marcus, C., Delattre, J.F., Marcus, C., CailliezTomasi, J.P., Palot, J.P., Ladam-Marcus, V., Menanteau, B. \& Flament, J.B. (1998) Right nonrecurrent inferior laryngeal nerve and arteria lusoria: the diagnostic and therapeutic implications of an anatomic anomaly. Review of 17 cases. Surg. Radiol. Anat., 20, 227-232.

Basterra, J., Dilly, P.N. \& Martorell, M.A. (1989) The autonomic innervation of the human vocal cord; neuropeptides. Laryngoscope, 99, 293-296.

Coady, M.A., Adler, F., Davila, J.J. \& Gahtan, V. (2000)
Nonrecurrent laryngeal nerve during carotid artery surgery: case report and literature review. J. Vasc. Surg., 32, 192-196.

Chan, W.F. \& Lo, C.Y. (2006) Pitfalls of intraoperative neuromonitoring for predicting postoperative recurrent laryngeal nerve function during thyroidectomy. World J. Surg., 30, 806-812.

Devèze, A., Sebag, F., Hubbard, J., Jaunay, M., Maweja, S. \& Henry, J.F. (2003) Identification of patients with a non-recurrent inferior laryngeal nerve by duplex ultrasound of the brachiocephalic artery. Surg. Radiol. Anat., 25, 263-269.

Fellmer, P.T., Böhner, H., Wolf, A., Röher, H.D. \& Goretzki, P.E. (2008) A left nonrecurrent inferior laryngeal nerve in a patient with right-sided aorta, truncus arteriosus communis, and an aberrant left innominate artery. Thyroid, 18, 647-649.

Gray, S.W., Skandalakis, J.E. \& Akin, J.T. Jr. (1976) Embryological considerations of thyroid surgery: developmental anatomy of the thyroid, parathyroids and the recurrent laryngeal nerve. Am. Surg., 42, 621-628.

Henry, J.F., Audiffret, J., Denizot, A. \& Plan, M. (1988) The nonrecurrent inferior laryngeal nerve: Review of 33 cases, including two on the left side. Surgery, 104, 977-984.

Hermans, R., Dewandel, P., Debruyne, F. \& Delaere, P.R. (2003) Arteria lusoria identified on preoperative CT and nonrecurrent inferior laryngeal nerve during thyroidectomy: a retrospective study. Head Neck, 25, 113-117.

Kato, A., Yamada, H., Yamada, T. \& Matsuura, T. (1996) Non-recurrent laryngeal nerve in three patients with thyroid cancer. J. Jpn. Bronchoesophagol. Soc., 47, 318-324. (in Japanese)

Kobayashi, M., Yuta, A., Okamoto, K. \& Majima, Y. (2007) Non-recurrent inferior laryngeal nerve with multiple arterial abnormalities. Acta Otolaryngol., 127, 332-336.

Maranillo, E., Vazquez, T., Quer, M., Niedenführ, MR., Leon, X., Viejo, F., Parkin, I. \& Sanudo, J.R. (2008) Potential structures that could be confused with a nonrecurrent inferior laryngeal nerve: an anatomic study. Laryngoscope, $\mathbf{1 1 8}$, $56-60$

Nagayama, I., Okabe, Y., Katoh, H. \& Furukawa, M. (1994) Importance of pre-operative recognition of the nonrecurrent laryngeal nerve. J. Laryngol. Otol., 108, 417-419.

Pemberton, J.D. \& Beaver, M.G. (1932) Anomaly of right recurrent laryngeal nerve. Surg. Gynec. Obstet., $\mathbf{5 4}$ 594-595.

Proye, C.A., Carnaille, B.M. \& Goropoulos, A. (1991) Nonrecurrent and recurrent inferior laryngeal nerve: a surgical pitfall in cervical exploration. Am. J. Surg., 162, 495-496.

Raffaelli, M., Iacobone, M. \& Henry, J.F. (2000) The "false" nonrecurrent inferior laryngeal nerve. Surgery., 128, 1082-1087.

Stedman, G.W. (1823) A singular distribution of some of the nerves and arteries in the neck and the top of the thorax. Edinb. Med. Surg. J., 19, 564-565.

Toniato, A., Mazzarotto, R., Piotto, A., Bernante, P., Pagetta, C. \& Pelizzo, M.R. (2004) Identification of the nonrecurrent laryngeal nerve during thyroid surgery: 20-year experience. World J. Surg., 28, 659-661.

Watanabe, A., Kawabori, S., Osanai, H., Taniguchi, M. \& Hosokawa, M. (2001) Preoperative computed tomography diagnosis of non-recurrent inferior laryngeal nerve. Laryngoscope, 111, 1756-1759. 